\title{
TRAMAS TEÓRICO-METODOLÓGICAS DEL TRABAJO SOCIAL EN EL CAMPO GERONTOLÓGICO
}

\section{THEORETICAL-METHODOLOGICAL PLOTS OF SOCIAL WORK IN THE GERONTOLOGICAL FIELD}

Fecha recepción: 23 de abril de 2019 / fecha aceptación: 24 de julio de 2019

Paula Mara Danel ${ }^{1}$ y Daniela Sala

Cómo citar este artículo:

Mara, P. y Sala, D. (2019).Tramas teóricos-metodológicos del trabajo social en el campo gerentológico. Revista Pensamiento y Acción Interdisciplinaria, 5(1), 77-94. DOI: http://doi.org/10.29035/pai.5.1.77

\section{Resumen}

El presente trabajo recorrerá ideas centrales para el análisis del campo gerontológico, desde la perspectiva relacional, presentando los ejes estructuradores del campo. Al mismo tiempo, en el artículo se procurará instalar los debates que el Trabajo Social desarrolla en torno a la dimensión crítica. En esa búsqueda, presentaremos las dimensiones que se hacen presentes en esa criticidad, asociadas a las ideas de Contexto, Práctica / experiencia /intervención, Género / generaciones, Narrativa / discurso, Saber/ poder y Producción de lo público.

El texto apela a hacer visibles nuestras paradojas, desarrolladas en los procesos interventivos del Trabajo Social en el campo gerontológico. La búsqueda es ponerle nombre a aquello que nos interpela y alojar a los sujetos que corporizan el paso del tiempo.

Palabras claves: campo gerontológico, Crítica, Trabajo Social, Narrativas, Sujetos

Abstract

The present paper will cover main ideas for the analysis of the gerontological field, from the relational perspective, presenting the structuring topics of the field. At the same time, the article will try to install the debates that Social Work develops around the critical dimension. In this search, we will present the dimensions that are present in that criticality, associated with the ideas of Context, Practice / experience / intervention, Gender / generations, Narrative /discourse, Knowledge / power and Production of the public.

Thetextinvokes to make visible our paradoxes, developed in the interventive processes of Social Work in the gerontological field. Thesearchis to namewhatinterpellateus and give place to the subjects that embody the passage of time.

Keywords: gerontological field, Critique, Social Work, Narratives, Subjects

1 Argentina, Dra en Trabajo Social (UNLP, 2016) Especialista en Epistemologías del Sur (Clacso 2019). Investigadora Adjunta de CONICET. Docente- investigadora del Instituto de Estudios en Trabajo Social y Sociedad (IETSyS) de la Facultad de Trabajo Social- UNLP. La Plata, Argentina. E-mail: danelpaula@hotmail.com

2 Argentina, Lic. en Trabajo Social, Especialista en Docencia Universitaria, Docente -investigadora del Instituto de Estudios en Trabajo Social y Sociedad de la Facultad de Trabajo Social (IETSyS) de la Facultad de Trabajo Social- UNLP. La Plata, Argentina. E-mail: saladaniela@gmail.com 


\section{Antecedentes Generales}

En el marco de nuestro recorrido como trabajadoras sociales vinculadas al campo de la vejez (desempeño en obra social nacional de personas mayores y en hogar de sostén y mantenimiento para mayores con diferentes grados de dependencia), como docentes de grado y posgrado (en materias relacionadas a la intervención profesional desde diferentes matrices teórico- metodológicas y en seminarios específicos de la temática) e investigadoras del campo gerontológico asociadas a los procesos de adscripción identitaria, presentaremos a continuación nuestras reflexiones sobre la intervención del Trabajo Social, desde perspectivas críticas en las particularidades del campo en cuestión.

Nos interesa desde estos múltiples espacios de producción de saberes, compartir los modos en que se traman de manera singular los aportes disciplinares / profesionales del Trabajo Social en un espacio tiempo asociado a las demandas de las personas mayores, y a los modos en que se estructuran las maneras de envejecer para este tiempo histórico y desde nuestro lugar del sur global.

\section{Tramando el campo gerontológico}

Nos interesa compartir desde la perspectiva relacional las ideas vinculadas al campo gerontológico, lo que supone recorrer un camino en el que se presenten debates y se anuden reflexiones que nos permitan comprender qué supone el campo gerontológico, qué límites produce y qué agentes interactúan en él.

Bourdieu (1990) nos señala que la idea de campo surge desde la necesidad de hacer inteligible lo social en momentos históricos en los que las sociedades se tornan más complejas. Desde esta búsqueda de enraizar en un espacio social singular las disputas que se desatan, dio lugar a la idea de campo.

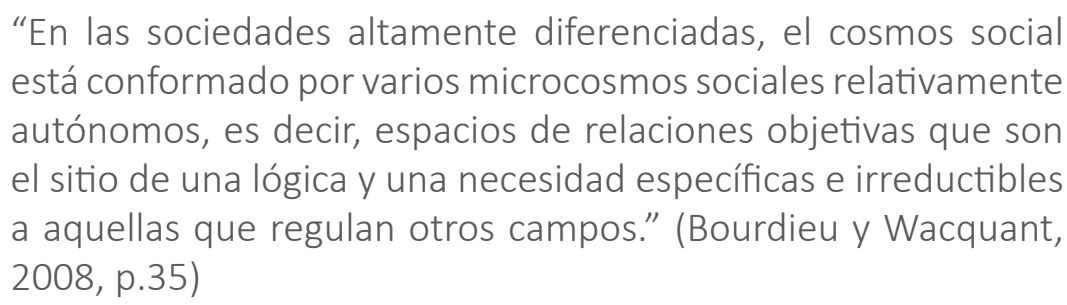

En tal sentido, si asumimos la mirada de Bourdieu en cuanto a que un campo supone una red o configuraciones de relaciones objetivas entre posiciones que tienen existencia más allá de sus ocupantes, nos surge la pregunta sobre qué particularidades tiene el campo gerontológico. ¿Cuáles son las posiciones en pugna?, ¿Qué capital específico en juego es el que motoriza las disputas? y ¿Cómo se produce la ilusión que permita la continuidad del juego? 
Una de las cuestiones que resulta interesante es anudar esta idea de campo con su analogía al juego y los ejes estructurantes del debate gerontológico. Hacemos referencia con la idea de ejes estructurantes a aquellos puntos neurálgicos que han sido tematizados por las disciplinas que confluyen en la gerontología. ¿Qué es la gerontología?

El consenso vigente señala que la gerontología asume como objeto al estudio de los procesos de envejecimiento, sociales e individuales, por lo que engloba dimensiones de orden físico, social y subjetivo de las personas mayores y los cambios que se producen en cada formación social en relación al envejecimiento de la población. Esta amplitud temática que asume la gerontología como objeto de indagación e intervención, tracciona la necesidad de que la misma sea de carácter interdisciplinario. Los ejes estructurantes de la gerontología las agrupamos en:

- Aquellos centrados en la dimensión biológico-funcional (Penny Montenegro y Melgar Cuellar, 2012)

- Los centrados en los cambios de orden subjetivo que se generan en el proceso de envejecimiento (Salvarezza, 1998)

- Los enfocados en los cambios de las intervenciones sociales frente al envejecimiento poblacional (intervención estatal, profesionales, las ofertas del mercado, etc) (Ludi, 2005 y Danel, 2008)

- Los centrados en el análisis pormenorizado de los cambios en los comportamientos de la población en torno a natalidad, mortalidad y migraciones. (Knopoff y Oddone, 1991)

- Los estudios que ponen en debate la dimensión simbólica, cultural de los modos de configurar la mirada social de la vejez. (Elder, 1974 y Yuni, 2015)

Estos posibles ejes que delinean los recorridos analíticos, nos brindan ideas en torno a qué disciplinas producen saberes que acumulan en cada eje estructurante y al mismo tiempo los transversalizan. Esa transversalización nos da pistas para pensar qué posiciones de esta red, intrínsecamente conflictiva, (campo gerontológico) son ocupadas por agentes profesionales que corporizan saberes disciplinares. Y al mismo tiempo supone reconocer los límites que el propio campo produce, lo que Bourdieu (1990) denominará efecto de campo. ¿Las personas mayores ocupan posiciones en el campo?, ¿Se trata de un campo que exclusivamente produce saber científico o genera espacio/lugar para los saberes de las personas mayores? Retomaremos hacia el final del texto este interrogante.

Siguiendo la idea de disputa, que supone la noción de campo gerontológico, y siempre en clave relacional, proponemos pensar cuáles son las determinaciones que las posiciones imponen a los agentes que las ocupan. ¿Qué regularidades se producen en el campo? Y allí es necesario pensar en clave de estructuración 
social de las edades y cómo eso genera prácticas y narrativas en torno al curso vital.

Disciplina y hedonismo se combinan en la medida en que las cualidades del cuerpo son entendidas como plásticas y los individuos son convencidos de asumir la responsabilidad por su propia apariencia (Debert, 2011, p.17).

La cita anterior nos da pistas para pensar cómo la estructuración social de las edades deja marcas en las expectativas sobre las trayectorias de los agentes, sobre las formas de trato social y las intervenciones estatales y del mercado. Bourdieu (1991) nos habla de cómo los habitus generan disposición a la percepción, pensamiento y acción, lo que podría ser enlazado a la idea de la disposición atravesada por la estructuración social de las edades. Por lo que la vida se periodiza y se producen constricciones sobre las vidas de todos/as los envejecientes.

Lo social hecho cosas y cuerpos, es vivido en tanto práctica social engendrada en el campo gerontológico. Los valores negativos asociados a las personas mayores (como cristalización de aquello a lo que no se desea llegar) se ponen en juego en "las cosas": los dispositivos de atención, los recursos asignados en forma prioritaria a la atención médica tradicional, entre otras, y en lo social hecho cuerpo a partir de los modos de percibir, lo pensable, lo decible y lo deseable en torno a los/as viejos/as (Danel, 2018, p. 95).

El campo gerontológico asume las constricciones y producen interrogantes que intentan desnaturalizar, pero cada tiempo histórico produce límites a aquello de lo que podemos dudar. ¿La mirada devaluada hacia las personas mayores es impugnada desde el campo gerontológico?

\section{Perspectivas críticas en el Trabajo Social}

Son muy diversas las influencias a las perspectivas críticas, desde el "trabajo social antirracista y multicultural; trabajo social antiopresor y antidiscriminativo; trabajo social feminista; diversas ramas de trabajo comunitario; trabajo social marxista; trabajo social radical; trabajo social estructural, y formas de investigación participativas y de acción." (Healy, 2001, p.14-15). Más allá de sus diferencias, la docente australiana plantea que comparten una orientación hacia la transformación radical donde existe un compromiso profesional con poblaciones oprimidas, cuestionando las estructuras de dominación y explotación, desde relaciones 
democráticas y participativas con los usuarios de los servicios en pos de acciones colectivas.

A continuación, desarrollaremos algunas ideas centrales de dos propuestas críticas que nos aportan para el análisis del campo gerontológico.

\section{Perspectiva histórico crítica: categorías centrales}

La perspectiva histórico crítica del Trabajo Social, cuyos exponentes principales son lamamoto $(1984,2003)$, Netto $(1997,2003)$, Montaño $(1998,1999)$ y Martinelli (1989, 2003), surge en Brasil en la década del '80, retomando los postulados del Movimiento de Reconceptualización, luego de cruentas dictaduras militares del Cono Sur. Basada en la teoría marxista, analiza al Trabajo Social y a la sociedad desde la perspectiva de totalidad, donde la totalidad es más que la suma de las partes y cada parte está atravesada por múltiples variables de esa totalidad.

En este sentido se plantea que el origen de la profesión no puede pensarse en forma endogenista, como evolución de anteriores formas de ayuda (filantropía y beneficencia), sino que su seguimiento se relaciona al momento en que el Estado comienza a dar respuestas a las manifestaciones de la Cuestión Social, fragmentándola y parcializándola en problemas sociales que serán abordados por Políticas Sociales y requieren de un nuevo agente que las implemente, en el marco del Capitalismo Monopolista.

De esta manera, para la perspectiva histórica crítica el/la trabajador/a social es un/a profesional asalariado/a, que vende su fuerza de trabajo a instituciones estatales, burguesas y aborda la reproducción material e ideológica de la clase trabajadora, en cuanto a sus necesidades y problemáticas manifestadas en la vida cotidiana, tanto en la asistencia directa con la población usuaria de las instituciones empleadoras, como en la gestión de recursos, atravesada también por una función educativa.

En este punto nos interrogamos: ¿sólo el TS aporta a esta reproducción o en realidad todas las profesiones configuradas en la modernidad producen prácticas reproductoras? Siendo que el TS aporta de este modo, nos preguntamos si la función educativa se desarrolla para reforzar esa reproducción o permite rupturas.

lamamoto $(1984,2003)$ plantea el carácter contradictorio del Trabajo Social, pues al mismo tiempo que se garantizan derechos para la clase trabajadora, se reproduce la estructura de clases y se genera consenso social, apaciguando conflictos. Esta cuestión podemos relacionarla con su concepto de autonomía relativa, donde el/la profesional si bien debe responder a las demandas de su empleador, tiene 
la posibilidad de elegir tanto su posicionamiento ético -político como el abordaje teórico- metodológico y operativo instrumental.

Volviendo a la perspectiva de totalidad, la categoría de mediaciones que trabaja Pontes (2004), nos brinda la posibilidad de superar lo aparente, fenoménico de la realidad que sería la instancia de la singularidad de una problemática, para relacionarla con lo universal, es decir aquellas leyes tendenciales que engloban a todas las problemáticas sociales en la sociedad capitalista y patriarcal en que vivimos, y de esta manera, abordarla en su particularidad. Esto permite un conocimiento más profundo de la situación, con sus múltiples atravesamientos, historizándola, teniendo en cuenta las causas y así desculpabilizar a los sujetos de sus condiciones, y colectivizar las demandas en pos de respuestas integrales del Estado.

En este marco es que se puede pensar el trabajo concreto con personas mayores, como una particularidad con sus características específicas. El/la trabajador/a social, además de su formación general, debe profundizar el conocimiento teórico acerca de las problemáticas que atraviesan a la población con la que trabaja (cuestiones demográficas del envejecimiento poblacional, características psicosociales de este grupo, servicios y prestaciones que se constituyen en respuestas institucionales) para pensar y llevar adelante sus estrategias de intervención.

\section{Otras criticidades del Trabajo Social}

Siguiendo los aportes de Teresa Matus, señalamos que el Trabajo Social se encuentra en debate profundo en pos de reconocer las tendencias críticas de la profesión y al mismo tiempo romper con la hegemonía de criticidad de la perspectiva histórico-crítica.

Esto supone el despliegue de otras categorías analíticas que permitan estructurar los modos en que el Trabajo Social produce su propia ruptura epistémica tendiente a configurar la criticidad. En ese punto, podemos afirmar que la crítica la entendemos como constitutiva del Trabajo Social, toda vez que algo del orden de la invención, de lo liminar ha sido propio de las ${ }^{3}$ trabajadoras sociales en los procesos interventivos. Con esto queremos señalar que en la historización de la profesión, (Gavrila, 2018; Scelsio, 2018; Danel, 2016, Travi, 2014) surge con perspicuidad que la ampliación de las agendas estatales, la consideración de los sujetos de la intervención, en tanto productores de saberes, ha sido una constante del Trabajo Social en su ejercicio profesional.

¿Cuáles son las dimensiones en las que se coloca esa criticidad anhelada desde las discusiones académicas y que viene siendo corporizada desde las intervenciones?

3 Se menciona a las profesionales del trabajo social en género femenino, ya que al tratarse de una profesión históricamente ejercida por mujeres se entiende que un acto de justicia es la generalización en femenino. 
Y allí coincidimos con Matus que "es preciso construir nuevas categorías conceptuales que permitan mostrar una realidad persistente y múltiple" (Matus: 2011, p 21), por lo que la criticidad en el Trabajo Social del siglo XXI se podría expresar en las siguientes dimensiones:

- Sujeto

- Contexto

- Práctica / experiencia /intervención

- género / generaciones

- Narrativa / discurso

- Saber/ poder

- Producción de lo público

\section{Sujetos y Contextos}

La idea de sujeto, que entendemos producen las perspectivas críticas del siglo XXI en el Trabajo Social, es la de complejidad, la de doble sujeción. Y al mismo tiempo, y desde el giro decolonial, la búsqueda de decolonizar el ser. El sujeto es comprendido no como categoría cerrada, sino como proceso de construcción continuo, con capacidad de acción transformadora.

Pensar la idea de sujeto, desde estas perspectivas incluye la idea de dimensión corporal. La existencia humana es corporal (Merleau-Ponty, 1962), lo que permite ir superando la separación cartesiana mente-cuerpo, donde se identifica al sujeto como exclusivo ser racional.

Siguiendo a Kesner, Edelman, Kordon y Lagos (2002), conceptualizamos a la subjetividad como construcción singular y colectiva, inacabada y producida de manera situada, histórica y socialmente. No podemos pensar la subjetividad por fuera del contexto en el que se desarrolla, por lo que las perspectivas críticas transitan las miradas sobre los sujetos

El habitus como sentido práctico, realiza la reactivación del sentido objetivado en las instituciones: producto del trabajo de inculcación y apropiación necesarios para que esos productos de la historia colectiva, que son las estructuras objetivas consigan reproducirse bajo la forma de disposiciones duraderas y ajustadas, que son condición de su funcionamiento, el habitus, que se constituye a lo largo de una historia particular imponiendo su lógica particular a la incorporación, y por el que los agentes participan de la historia objetivada en las instituciones, es lo que 
permite habitar las instituciones, apropiárselas prácticamente y de este modo mantenerlas vivas, activas, vigorosas, arrancarlas continuamente del estado de letra muerta, de lengua muerta, hacer revivir el sentido que se encuentra depositado en ellas, pero imponiéndoles las revisiones y transformaciones que son la contrapartida y condición de la reactivación (Bourdieu, 1991 p 99).

Pensando junto a Bourdieu, el sujeto toma rostro de agente social que transita lo social hecho cuerpo y hechos cosas, produciendo y reproduciendo aquello que habita. Siguiendo esta lógica, planteamos que las personas mayores resultan ser sujetos que se siguen construyendo, por un lado, por el cambio que se genera en los roles sociales asignados y asumidos, los vínculos que se dejan y que se generan, el cambio de contextos por los que transitan. Las personas mayores van produciendo un cambio subjetivo que puede ser vivenciado como angustiante, liberador y otras múltiples formas.

\section{Intervención profesional: prácticas o experiencias.}

En relación a la idea de práctica profesional, creemos que la misma ha devenido hegemónicamente en la categoría de intervención. Y en tanto, tal no ha dejado lugar a la idea de experiencia, ya que la misma se enlaza a la idea de reiteración de tareas.

\footnotetext{
"No importan cuántos años lleve alguien haciendo algo, puede sencillamente, cometer el mismo error muchas veces... De allí que ser bueno en algo, no consiste en hacerlo muchas veces sino en comprenderlo en su sentido y abrirlo a la duda de su rutinización" (Matus, 2011,p.23).
}

Entendemos que la idea de experiencia en tanto posibilidad de comprender el sentido de la acción y abrirlo a la duda, es constitutiva a la idea de intervención generada desde las perspectivas críticas.

En la última década, y concordante con los debates de las ciencias sociales en general, el Trabajo Social incluye la opción decolonial en la búsqueda de comprensión desde desprendimientos epistémicos a los sujetos de la práctica, en tanto producto de la colonialidad del saber, poder y del ser. Siguiendo a Carballeda, (2018) esos sujetos de la práctica encarnan los efectos de esa colonialidad en un contexto que instala discursos individualistas y meritocráticos. 
La idea de experiencia en diálogo con los debates de la intervención, potencia análisis e intervenciones en lo social y permite al Trabajo Social incrementar posibilidades.

De allí que este concepto de experiencia nos abre en forma inequívoca a explorar las relaciones entre discursos y prácticas sociales. Estos dos focos permiten iluminar una tercera cuestión en el tratamiento del discurso: el modo en que se determine lo real, más el enlazamiento con las prácticas sociales, se jugarán dando resultados muy distintos en la forma de nombrar al otro discurso. Así al usar este concepto de experiencia, el otro se vuelve otro reconocidamente circunscrito (Matus, 2011, p. 24).

El enfoque narrativo en educación (Contreras, 2010) propone que la experiencia es la que pone en movimiento el pensamiento. Por lo que produce un tipo de saber que supera la separación dualista entre teoría y práctica.

Pensar desde la idea de experiencia, posibilita comprender la configuración de lo social,

donde las configuraciones de lo social desbordan las formas clásicas de interpretarlas, donde el proceso reductivo de la dialéctica de la modernización, nos enfrenta a trabajar con sus rostros duros, con sus fragmentos, con sus opacidades, con sus espectáculos, es imperioso encontrar nuevas formas para nombrar lo que allí sucede, ya que, además, para el Trabajo Social estos desafíos se presentan corporalmente con nombres y apellidos. La propuesta es colocar nuevos mapas de la experiencia en Trabajo Social, como respuesta a esa exigencia de realizar una comprensión social compleja, donde se rescate la posibilidad humana de hacer emerger no sólo la evidencia de las contradicciones sociales, sino de colocar en el mundo, cosas que nunca existieron (Matus, 2011, p.31).

La intervención profesional, pensada desde la idea de experiencia, permite la constelación de saberes que con lenguajes múltiples puedan nominar, y generar esfuerzos por poner en diálogo las grietas entre los discursos expertos y la vida cotidiana de los sujetos. La intervención permite desnaturalizar y problematizar situaciones para llevar a cabo una praxis social superadora.

En la intervención con personas mayores, la categoría de experiencia cobra múltiples sentidos. Por un lado, cuando se recuperan las experiencias de vida de las mismas, desde protagonismos y con la posibilidad de generar experiencias que generen algo novedoso, que quieran implicarse, ser realmente protagonistas de sus vidas, más allá de los apoyos que puedan requerir para llevarlo a cabo. La 
intervención con mayores, en línea con lo que venimos señalando supone aportar a la desnaturalización y problematizar situaciones vitales en pos de generar una praxis social superadora.

\section{Intersecciones: género y generaciones}

Si bien en apartados anteriores hemos hecho referencia a la gerontología como el estudio de los procesos de envejecimiento y los diferentes ejes en los cuales hacen foco, resulta oportuno analizar el envejecimiento poblacional desde una perspectiva de género, en pos de aportar a la construcción de una ciencia no androcéntrica, tanto en las teorías científicas con sus categorías, como en el proceso de investigación, donde las mujeres y sus formas de hacer ciencia no sean infravaloradas.

El género como categoría analítica, es una construcción socio histórica que establece relaciones de poder binarias y asimétricas, transversal a todos los ámbitos sociales. Designa al conjunto de prácticas, símbolos, representaciones, normas y valores que la sociedad elabora a partir de diferencias anatómicas que dan sentido a relaciones entre personas (Teresita de Barbieri, 1994, citada por Roberto Inda, 2006). Las marcas de género se adquieren durante la socialización.

Al referirnos a la interseccionalidad retomamos lo postulado por Raquel (Lucas) Platero Méndez (2014), donde diferentes fuentes estructurales de desigualdad u organizadores sociales, como son género, edad, clase social, etnia y orientación sexual, mantienen relaciones recíprocas, pudiendo pensarlo como una matriz de dominación. Por lo tanto, el campo gerontológico no puede ser abordado en forma aislada de su contexto y múltiples atravesamientos, sino que debe incluir la heterogeneidad de vejeces presentes.

En ese sentido, identificar a nuestras ancestras (Navarro, 2017), como quienes nos anticiparon en la lucha por los derechos de las mujeres, y a partir de su legado continuar, profundizar, generar rupturas con el patriarcado y la meritocracia capitalista.

El cruce generacional y la mirada deshecha del género (no binaria) se constituyen en dimensiones centrales en las perspectivas críticas del Trabajo Social del Siglo $X X I$. 


\section{Narrativas y discursos}

Las narrativas nos permiten adentrarnos a las formas en que se producen sentidos a partir de lo que las personas dicen, de sus modos de simbolizar y transmitir mediante las expresiones verbales, sus teorías sobre aquello que les acontece, las relaciones con otros sujetos y la posibilidad de tomar decisiones sobre sus propias trayectorias vitales. Las narrativas nos permiten establecer relaciones con las biografías personales y el lugar otorgado a aquello que los hace vincular al trabajo social.

Pensar en clave de narrativa como portador de significados, de saberes, nos vincula con los sujetos de la intervención como productores de saberes. Esta afirmación constituye un salto cualitativo en cuanto que permite desplazarse, correrse, de relaciones paternalistas y de reconocimiento de falencias e incompletudes. Especialmente evitar la infantilización que muchas veces se produce en la intervención con personas mayores.

La idea de narrativas y discursos, como modos singulares de producción de las voces en primera persona (voz subjetivante), aquellos textos que se producen en el campo gerontológico y en el Trabajo Social (voz objetivante) que busca producción certera / académica de aquellos que caracteriza a la vejez en general y a la intervención en lo social. La polifonía de voces, surgida de textos caracterizada por la inclusión de voces diversas.

Esta idea de narrativas y discursos produciendo subjetiva, objetiva y polifónicamente es recuperada desde miradas interdisciplinarias en el cruce entre la comunicación (Martín Barbero, 2003) y las discusiones sobre la producción de hegemonía (Laclau, 2005).

\section{Saber / poder}

Las narrativas de los sujetos de la intervención, sumado a las formas en que el Trabajo Social produce saber y la legitimidad de la intervención, hace necesario que se discuta, se tematicen las relaciones de poder que suponen las posiciones diferenciales.

Siguiendo lo planteado por Foucault $(1999,2002)$ el poder no es algo que posea determinado actor social, sino que está distribuido en diferentes puntos de la malla social y se ejerce en la lucha por la palabra autorizada, en este sentido se plantea la relación entre saber y poder, pues quien tenga mayor información y acceso a conocimientos socialmente valorados, podría ejercer mayor poder.

El positivismo generó la división de la realidad en áreas de conocimiento donde los expertos (especialmente hombres, blancos, adultos, occidentales, racionales, 
objetivos) son los únicos que aparentemente pueden estudiar, definir, clasificar, realizar indicaciones de intervenciones, que resultan normalizadoras sobre otros que deben aceptar con pasividad su situación. Un ejemplo claro de esto es la postura del modelo médico hegemónico.

También se debe analizar las relaciones de poder en el Trabajo Social (Falla Ramírez, 2016), en la realización de entrevistas institucionales y domiciliarias, para llegar a construir dictámenes, diagnósticos sociales, determinación de prioridades, donde la persona mayor puede estar en una situación y relación de subordinación o configurar conjuntamente resistencias al poder hegemónico desde prácticas de libertad, ya que los valores que sustentan la intervención no son neutros.

\section{Producción de lo público}

Nos resulta sugerente señalar que desde las perspectivas críticas del Trabajo Social en el Siglo XXI, cuando se analizan las posiciones estatales frente a determinados problemas, interesa pensar los modos en que las políticas (públicas o sociales) amplían el espacio de lo público.

Las políticas constituyen un conjunto encadenado de decisiones y acciones que son resultado de las interacciones estructuradas y repetidas entre diferentes actores, que por diversas razones, están implicados en el surgimiento, la formulación y la resolución de un problema, políticamente definido como público (Subirats, 2008)

"Lo público está siendo estructurado (Bourdieu, 1991), por lo que se produce en proceso y resulta inacabado. En lo público, en las dimensiones de espacio y tiempo, se concentran y organizan las cualidades de lo colectivo, de coproducción. Retomando los clásicos estudios de Lefevre (1991), el espacio lo comprendemos como una producción social, llevada a cabo entre desconocidos y basada en interacciones efímeras. El tiempo, está también estructurado por lo que existen modos de estructuración de las edades, que suponen directrices y moldeamientos." (Danel, 2019).

Lo público, que es de todos y todas, que pulsa aquello del orden contextual resultante subjetivo, se torna central y organizador del despliegue interventor del Trabajo Social.

Resulta relevante analizar las prioridades establecidas socialmente para la asignación de recursos, la formulación de las políticas sociales, abordando problemáticas en forma parcial y la incorporación o no de la perspectiva de las personas mayores en las temáticas que los atañen. 


\section{Resultados y Discusión}

El Trabajo Social contemporáneo del siglo XXI, requiere reforzar y sostener el esfuerzo intelectual, para comprender las relaciones entre debates categoriales de la profesión, experiencia e intervención. Asumir nuestra tradición liminar, de interpelación, de búsqueda en ampliar los campos de los posibles.

"Una experiencia contemporánea de la intervención social, debe encarar frontalmente la existencia de estas posturas involucradas, a veces, en el propio entramado de la intervención (Matus, 2011, p.37)

Debido a que la experiencia se vivencia y supone una novedad, generando reflexivamente una ruptura con formas de pensamiento anteriores, genera la necesidad de categorías nuevas que nos permitan pensarla. La experiencia genera saberes situados, que no pueden ser traspolados o reproducidos mecánicamente sino que "Lo que aprendemos de la experiencia es la posición a partir de la que afrontamos nuevas situaciones... como modo de mirar, de estar, de interpretar, de actuar" (Contreras, 2010, p. 33)

Por eso la experiencia nos interpela, y apostar al ejercicio profesional crítico en el campo gerontológico, conlleva preguntarnos sobre la posibilidad de expresión de necesidades y deseos de las hombres y mujeres mayores, en un contexto donde el consumismo apunta a desechar lo viejo en búsqueda de lo novedoso, lo cual incluye mercancías, pero también sujetos. Las personas mayores con autonomía son vistas como posibles consumidoras de bienes y servicios cuyas publicidades crean la ilusión de retroceder el paso del tiempo, como las cremas antiage; en cambio la población que presenta algún deterioro físico o cognitivo es la meta de la industria farmacológica y de empresas que brindan servicios de atención domiciliaria o institucional, medicalizando procesos propios del curso vital.

Entonces nos preguntamos: ¿de qué manera desde el Trabajo Social podemos poner en la agenda pública sus derechos sin arrogarnos la capacidad de definirlos ni homogeneizar sus experiencias?

Nos interesa que la intervención profesional del Trabajo Social en el siglo XXI, sea capaz de anudar la criticidad en el campo gerontológico, asumiendo que en los distintos cursos vitales, las heterogeneidades se hacen presentes, toman rostro, construyen narrativas y nos invitan a pensar, a soñar y a comprometernos con una sociedad para todas las edades.

Finalmente, nos interesa volver a interrogarnos sobre la posición que ocupan las personas mayores en el campo gerontológico, cómo se alojan sus saberes, como las diferenciales de género son atendidas en el campo. Señalamos que las personas mayores son pensadas como objeto de consumo para mitigar las marcas del paso del tiempo, y cuando esas marcas se expresan en limitaciones, se consolida ese lugar pasivo de consumo. 


\section{Conclusiones}

Posicionarnos desde perspectivas críticas, implica desnaturalizar las relaciones de poder donde determinados sujetos tienen la capacidad de decidir sobre otros, es comprender las disputas dentro del campo gerontológico por definir categorías, formas de abordaje; donde la voz de los sujetos involucrados no sólo sea escuchada, sino reconocida como válida y produzca cambios en la política pública. Identificar a las personas mayores como ciudadanos y sujetos de derecho equivale a no priorizar el cuidado sobre su participación, con protagonismo y desde sus propios deseos.

Tomamos el desafío planteado por lamamoto de ser profesionales propositivas, para lo cual tenemos que desarrollar la "capacidad de descifrar la realidad y construir propuestas de trabajo creativas y capaces de preservar y tornar efectivos los derechos, a partir de las demandas emergentes en el cotidiano" (Iamamoto, 2003, P 33) Esto se puede llevar adelante tanto, denunciando los cambios regresivos en las Políticas Sociales, difundiendo circuitos de acceso a los programas sociales e impulsando estrategias organizativas en la reivindicación de derechos vulnerados.

Hacer visibles nuestras paradojas, ponerle nombre a aquello que nos interpela y alojar a los sujetos que corporizan el paso del tiempo.

\section{Referencias}

Abad Miguélez, B. (2016). La Producción Socio-Institucional de Sufrimiento Social. International and MultidisciplinaryJournal of Social Sciences 5 (1), 1-25. https://doi.org/10.17583/rimcis.2016.1802.

Barbero, M (2003). Comunicación y cultura: una relación compleja. En: Portal Moreno R y Recio Silva (Comp) Comunicación y comunidad. La Habana: Félix Varela.

Bourdieu, P., \& Wacquant, L. (2008). Una invitación a la sociología reflexiva (2a ed.). Buenos Aires: Siglo XXI.

(1990). Algunas propiedades de los campos. En Sociología y cultura. México: Grijalbo.

(1991). El sentido práctico. Madrid: Taurus.

Carballeda, A (2018). (Coord.), Las sociedades de la desigualdad. Entredichos. Intervenciones y Debates en Trabajo Social, Dossier N4. Recuperado de 
http://entredichos.trabajosocial.unlp.edu.ar/2018/12/04/n4-lassociedades-de-la-desigualdad/

Contreras Domingo, J., Pérez de Lara, N., \& Arévalo Vera, A. (2010). La experiencia y la investigación educativa. Madrid: Morata.

Danel, P. (2008) Adultos mayores institucionalizados: Objetos de protección, cuidado y rentabilidad [En línea]. Jornadas de Cuerpo y Cultura de la UNLP, 15 al 17 de mayo de 2008, La Plata. Recuperado de www.fuentesmemoria. fahce.unlp.edu.ar/trab_eventos/ev.654/ev.654.pdf

-(2016) Trabajo Social y Discapacidad. Intervenciones, trayectorias, temporalidades. Paraná: Editorial La Hendija.

------- (2018) De disputas y hegemonías en las intervenciones con personas mayores en espacios comunitarios. Revista Intervenciones, 8(2), 92-108. recuperado de https://intervencion.uahurtado.cl/index.php?journal=intervencion

- (2019) Envejecimiento en contextos de desigualdad: tensiones entre redistribución y restricciones. En Segundo Seminario Evidencias del Trabajo Social en Gerontología. UNAM (En Prensa)

Elder, G. (1974) Children of thegreatdepresion. Chicago: University of Chicago Press.

Falla Ramírez, U. (2016). La intervención como forma de poder en el trabajo social. Tabula Rasa, (24), 349-368. https://doi.org/10.25058/20112742.69

Foucault, M (1999) Vigilar y Castigar. Nacimiento de la prisión. México: Siglo XXI.

Foucault, M (2002) Historia de la sexualidad. Buenos Aires: Siglo XXI Editores.

Gavrila, C. (2018). Hermosear y vigilar Las Visitadoras de Higiene Social de la Universidad Nacional de La Plata en el proceso de institucionalización del trabajo social en la Argentina (1922-1948) (Tesis de maestría). Buenos Aires: Universidad Nacional de La Plata. Recuperado de http://sedici.unlp.edu.ar/ handle/10915/70919

Debert, G (2011). La reinvención de la vejez. Buenos Aires: Siglo XXI.

Healy, K (2001). Trabajo social: perspectivas contemporáneas. Madrid: Morata.

lamamoto, M. (2003). El Servicio Social en la Contemporaneidad. Trabajo y formación profesional. São Paulo: Cortez.

lamamoto, M. \& Caravallo (1984). Relaciones sociales y Trabajo Social. Esbozo de interpretación histórico-metodológica. Lima: Alfa. 
Inda, N. (2006). La perspectiva de género en investigaciones sociales. En Verschuur C. \& Hainard, F. Des brèchesdans la ville, 37-54.

Knopoff, R., Oddone, M., \& Daino, L. (1991). Dimensiones de la vejez en la sociedad argentina. Buenos Aires: Centro Editor de América Latina.

Kordon, D., Edelman, L., Lagos, M. \& Kersner, D. (2002). Abordaje clínico y rol profesional. En Kersner, D., Jorge, M. \& Madariago, C. (Coords.). Paisajes del dolor, senderos de esperanza. Salud mental y derechos humanos en el Cono Sur. Buenos Aires: Polemos.

Laclau E (2005). La razón populista. Buenos Aires: Fondo de Cultura Económica.

Laclau, E. (2002). Misticismo retórica y política. Buenos Aires: Fondo de Cultura Económica.

Lefevre, H. (1991). Theproduction of space. Oxford: Blackwell.

Ludi, M (2005). Envejecer en un contexto de (des) protección social. Buenos Aires: Espacio.

Matus, T. (2011). Experiencia y pobreza en el trabajo social: una lectura frankfurteana. Questão. Relecturas De Servico Social Contemporaneo, 13 (24), 19-42.

Melgar Cuellar, F. \& Penny Montenegro, E. (2012). Geriatría y gerontología para el médico internista. Bolivia: La hoguera investigación. Recuperado de www.smiba.org.ar/archivos/geriatria_gerontologia.pdf.

Merleau-PontyM.(1962). Phenomenologyfperceotion. New York: HuamnitiesPress.

Montaño, C. (1998). La naturaleza del Servicio Social: un ensayo sobre su génesis, especificidad y su reproducción. São Paulo: Cortez.

- (1999). El Servicio Social frente al Neoliberalismo. Cambios en su base de sustentación funcional-laboral. Boletín electrónico Surá 41, 1-30.

Navarro M. (2017). Anudamientos teóricos y herramientas analíticas en investigaciones sobre envejecimiento, género y salud. En Actas digitales de Il Congreso Latinoamericano de Teoría Social y Teoría Política "Horizontes y dilemas del pensamiento contemporáneo en el sur global", Buenos Aires, 2 al 4 de Agosto de 2017. Recuperado de http://diferencias.com.ar/congreso/ ICLTS2015/wp/index.php/mesas-y-ponencias-2017/.

Netto, J. P. (1997). Capitalismo Monopolista y Servicio Social. São Paulo: Cortez. 
Netto, J. P. (2003). La construcción del proyecto ético-político del Servicio Social frente a la crisis contemporánea. En Borgianni, E.; Guerra, Y. \& Montaño, C. (Orgs.) Servicio Social Crítico. São Paulo: Cortez

Platero Méndez, R (Lucas). (2014).Metáforas y articulaciones para una pedagogía crítica sobre la interseccionalidad. Revista Quaderns de Psicología 16,1, 55-72

Pontes, R (2004). Mediación: categoría fundamental para el trabajo del asistente social. En Borgianni, E. \& Montaño, C. Servicio Social crítico. Hacia la construcción del nuevo proyecto ético-político profesional. São Paulo: Cortez.

Salvarezza, L. (1998). La vejez. Buenos Aires: Paidos.

Scelsio, J. (2018). Higienismo e intervención social en la ciudad de La Plata. Su relación con el origen profesional de las Visitadoras de Higiene Social de la UNLP en el año 1938. Tesis de Maestria. Recuperado de http://sedici.unlp. edu.ar/handle/10915/73327.

Travi, B. (2014). Investigación histórica e identidad en trabajo social. Nuevas y renovadas epistemologías para los nuevos tiempos. Revista del Departamento de Ciencias Sociales, 5, 37-58.

Yuni, J (2015). Perspectivas críticas acerca de la construcción social de la vejez y las intervenciones socio-culturales. En: Danel, Paola y Tordó (2015) Más mayores, más derechos. Diálogos interdisciplinarios sobre vejez. La Plata: Edulp. Recuperado de www.editorial.unlp.edu.ar/uploads/docs/gerontologia.pdf. 
Dirección de correspondencia:

Paula Mara Danel

Doctora en Trabajo Social, UNLP.

Académica Universidad Nacional de la Plata

Contacto: danelpaula@hotmail.com. 